\title{
Encontrando soluciones sostenibles con pequeños productores de papa a través de investigación participativa en la sierra centro de Ecuador
}

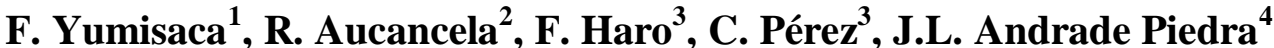

RESUMEN

Con el propósito de generar alternativas para la producción de papa en zonas de altura a través de Investigación Participativa (IP), se conformaron dos Comités de Investigación Agrícola Local (CIAL) en las comunidades Belén y Cotojuan de la provincia de Chimborazo, Ecuador. Se identificaron como principales limitantes al tizón tardío (Phytophthora infestans), bajo rendimiento, pérdida de calidad para fritura de la variedad INIAP-Fripapa y deficiente acceso a mercado. En los ensayos de prueba y comprobación, las variedades nativas Puca Shungo, Yana Shungo y Chaucha Roja se identificaron como las mejores por el rendimiento alcanzado y su potencial de mercado. Adicionalmente, se hicieron acercamientos con Fritolay para iniciar pruebas de procesamiento para hojuelas de colores. En paralelo, se desarrolló un Enfoque Participativo de Cadenas Productivas (EPCP) en la ciudad de Riobamba, a través del cual se determinó la aceptación de Chaucha Roja y Yana Shungo para platos gourmet, generando una demanda constante. El trabajo realizado permitió desarrollar un proceso de IP para dar respuesta a una problemática concreta en la zona y muestra la complementariedad entre las metodologías de CIAL y de EPCP.

Palabras clave: Acceso a mercado, economía agrícola.

\section{Sustainable alternatives for small potato producers in the highlands of Ecuador through participatory research}

\begin{abstract}
Two Local Agricultural Research Committees (LARCs) were implemented in the communities of Belén and Cotojuan (province of Chimborazo) with the purpose of generating alternatives for small potato farmers in the high Andes of Ecuador. Main problems were late blight (Phytophthora infestans), low yields, low frying quality of the potato cultivar INIAP-Fripapa, and poor market access. In the trials, the native varieties Puca Shungo, Yana Shungo and Chaucha Roja were identified as the best, because of yield and market potential. Samples of these varieties were sent to the multinational company Fritolay to test their frying quality for colored chips. In parallel, a Participatory Market Chain Approach (PMCA) was implemented in the city of Riobamba, generating a constant demand for the varieties Chaucha Roja and Yana Shungo in a gourmet restaurant. This work allowed implementing a Participatory Research process to provide answers to a specific demand and show the complementarities between the LARC and the PMCA methodologies.
\end{abstract}

Key words: market access, agricultural economy.

\footnotetext{
${ }^{1}$ Investigador, Instituto Nacional Autónomo de Investigaciones Agropecuarias (INIAP), Programa Nacional de Raíces y Tubérculos rubro papa (PNRT-Papa), Riobamba, Ecuador. fyumisacaj@yahoo.com

2 Asistente de campo, INIAP, PNRT-Papa, Riobamba, Ecuador.

${ }^{3}$ Asistentes de investigación, proyecto InnovAndes, Riobamba, Ecuador.

${ }^{4}$ Investigador, Centro Internacional de la Papa (CIP), Quito, Ecuador.
} 


\section{Introducción}

En el Ecuador, las papas nativas tienen una presencia comercial limitada en el mercado, debido principalmente al desconocimiento por los consumidores urbanos y a la amplia cobertura que han alcanzado las variedades mejoradas (Monteros et al., 2005). Sin embargo, la tendencia actual de los hábitos de consumo, la valoración de la calidad, la conciencia creciente de la defensa de los derechos del consumidor y la conservación del medio ambiente y la biodiversidad, pueden ofrecer nuevas oportunidades de mercado para las papas nativas, ya sea en forma natural o procesada (Ordinola et al., 2007). Estas nuevas oportunidades de mercado han sido aprovechadas en los Andes a través del Enfoque Participativo de Cadenas Productivas (EPCP) (Bernet et al., 2006; López et al., 2010).

Una metodología que puede complementar al EPCP es el Comité de Investigación Agrícola Local (CIAL). El CIAL es un equipo de investigación local que pertenece a la comunidad, conformado y orientado por la misma, permitiendo a los agricultores relacionarse con el sistema de investigación formal para tener acceso a nuevas destrezas, información y tecnologías que puedan ser útiles a nivel local (Ashby et al., 1999). Un CIAL puede constituirse en el brazo técnico de una organización de productores, que investigue en función de las demandas reales del mercado (Thiele y Bernet, 2005).

El objetivo del presente trabajo fue implementar alternativas para la producción de papa en zonas de altura a través de Investigación Participativa (IP), específicamente mediante CIALs.

\section{Materiales y métodos}

Este trabajo se llevó adelante entre 2007 y 2010. Se realizó un acercamiento a las comunidades Cotojuan y El Belén ubicadas en el cantón Colta, provincia de Chimborazo, Ecuador, quienes se mostraron interesadas en conectarse a mercados de alto valor de papa. Luego en asambleas comunitarias se conformaron CIALs con la participación de entre 12 y 20 productores/as en cada comunidad. A través de un Diagnóstico Rural Participativo (DRP) se identificaron las principales limitantes del cultivo de papa, luego se priorizaron posibles soluciones y se planificaron ensayos con tecnologías promisorias disponibles, generadas por el Programa de Raíces y Tubérculos rubro Papa del Instituto Nacional Autónomo de Investigaciones Agropecuarias (INIAP). Se implementaron ensayos de prueba en lotes comunitarios, se realizaron evaluaciones participativas en la etapa de floración y cosecha. Además se hicieron pruebas de degustación en papa cocida y en procesado como hojuelas. Se entregaron los resultados a la comunidad y se implementaron ensayos de comprobación y luego parcelas de producción. En paralelo, se desarrolló un proceso de EPCP con papas nativas en la ciudad de Riobamba (López et al., 2010) que busco generar innovaciones a partir de las papas nativas.

\section{Resultados y discusión}

En el DRP se identificó que las principales limitantes en el cultivo de papa eran la presencia de tizón tardío o lancha (Phytophthora infestans), bajo rendimiento, y pérdida de calidad de la variedad INIAP-Fripapa debido a que, cuando se siembra sobre los $3300 \mathrm{~m}$ de altitud, pierde características para procesamiento de fritura, lo cual determina disminución en su precio y dificulta su acceso al mercado.

En los ensayos de prueba (primer ciclo de IP, 2007-2008) se evaluó la adaptación agronómica de las variedades nativas: Chaucha Amarilla y Chaucha Roja, y de los clones mejorados a partir de variedades nativas: 05-16-6 (localmente conocida como Puca Shungo) y 05-19-3 (localmente conocida como Yana Shungo). En floración se determinó que los criterios más importantes para los productores son: resistencia a tizón tardío, tallos gruesos, planta vigorosa (con buena cobertura) y altura de planta. En base a estos criterios se ubicaron en orden de priorización: Puca Shungo, Yana Shungo, Chaucha Amarilla y Chaucha Roja. En la cosecha, los criterios más importantes fueron: 
producción, color de la cáscara, color de la pulpa y buen sabor. De acuerdo a estos criterios, el orden que alcanzaron las variedades fueron: Chaucha Roja, Yana Shungo, Chaucha Amarilla y Puca Shungo. Los rendimientos promedio que alcanzaron las variedades en estudio en las dos localidades fueron: Puca Shungo 29,9 t/ha, Yana Shungo 24,8 t/ha, Chaucha Amarilla 20,5 t/ha y Chaucha Roja 18,6 t/ha. En la reunión de entrega de resultados a la comunidad, se tomó la decisión de continuar evaluando en el siguiente ciclo de comprobación las variedades Chaucha Roja, que en la degustación de cocido fue la mejor, y Yana Shungo y Puca Shungo que resultaron ser las mejores para procesamiento en hojuelas, ya que además de presentar buena calidad de fritura tienen la pulpa de color morado oscuro y rojizo, respectivamente, lo que resultó ser atractivo para los consumidores.

En el siguiente ciclo de IP (2008-2009), o etapa de comprobación, se implementaron ensayos con cada productor del CIAL Cotojuan y se obtuvieron rendimientos promedio de 16,3 t/ha con Puca Shungo, 13,6 t/ha con Yana Shungo y 6 t/ha con Chaucha Roja. En este ciclo se presentaron condiciones de sequía y heladas, que afectaron en mayor proporción a la variedad Chaucha Roja, la cual disminuyó considerablemente su rendimiento. Adicionalmente, se hicieron acercamientos con la empresa Fritolay para iniciar pruebas preliminares de procesamiento para hojuelas de colores, para lo cual se entregó 26 qq de Puca Shungo y 23 qq de Yana Shungo. De estas primeras pruebas se rechazó un $40 \%$ de las muestras ya que los niveles de azúcares reductores fueron muy altos, evidenciándose que se necesitaba generar mayor información sobre el manejo agronómico de estos materiales en cuanto se refiere a épocas de cosecha y altitud.En paralelo, a través del EPCP se determinó que las variedades Chaucha Roja y Yana Shungo fueron las mejores para platos tipos gourmet en un restaurant de esta ciudad, generando una demanda constante de 4 qq de Chaucha Roja y 1 qq de Yana Shungo por semana.
En el tercer ciclo de producción comercial (ciclo IP, 2009-2010), se implementaron parcelas individuales con los productores participantes en base a la demanda real y se instalaron además ensayos con Puca Shungo y Yana Shungo para evaluar el efecto de cuatro épocas de cosecha y altitud en la calidad de la fritura.

\section{Conclusiones}

Como fruto de este trabajo se obtienen las siguientes conclusiones: (i) la metodología CIAL, permitió desarrollar un proceso de IP, para dar respuesta a una problemática concreta en la zona, proponiendo de manera conjunta con los productores soluciones sostenibles $\mathrm{y}$ complementando al EPCP; (ii) las alternativas tecnológicas, en este caso genotipos de papa, son adoptadas si se puede obtener buenos resultados a nivel agronómico y sobre todo si son promisorios para nichos de mercados especializados que permitan mejores alternativas a los pequeños productores; y (iii) se comprobó el potencial de aceptación que pueden presentar las papas nativas para nichos específicos de mercado, como en este caso los restaurantes gourmet.

\section{Agradecimientos}

Este trabajo se realizó con fondos de la Agencia de Desarrollo y Ayuda Internacional de Nueva Zelandia (NZAid) a través del proyecto "Fortalecimiento de las capacidades para innovar y reducir la pobreza en los Andes, InnovAndes". EL EPCP realizado en Riobamba fue implementado por la Fundación Minga para la Acción Rural y la Cooperación (M.A.R.CO.), en alianza con el Consorcio de Pequeños Productores de Papa (CONPAPA) y el INIAP, con financiamiento del proyecto Alianza Cambio Andino.

\section{Literatura citada}

Ashby, J.A., Braun, A., Brekelbaum, T., Gracia, T., Guerrero, M.P., Quirós, C.A., Roa, J.I. 1999. Investing in Farmer Researchers: Experience in Latin America. Centro Internacional de Agricultura Tropical (CIAT). Cali, Colombia. 
Bernet, T., Thiele, G., Zschocke, T. 2006. Participatory Market Chain Approach (PMCA) User Guide. Primera Edición. Centro Internacional de la Papa (CIP), Lima, Perú. 169 p.

López, G., Oros, R., Rodríguez, F., López, J, Manrique, K. 2010. Contribución del EPCP al mercadeo de las papas nativas en los Andes. Revista de la Asociación Latinoamericana de la Papa 15: 94-96.

Monteros, C., Cuesta, X., Jiménez J., López, G. (eds). 2005. Las papas nativas en el Ecuador. Primera Edición. Programa Nacional de Raíces y Tubérculo Rubro Papa/FORTIPAPA. Instituto Nacional Autónomo de Investigaciones Agropecuarias (INIAP). Quito, Ecuador. 32 p.
Ordinola, M. Bernet, T., Manrique, K. 2007. T'ikapapa: Vinculando consumidores urbanos y Pequeños Productores Andinos con la Biodiversidad de la Papa. Proyecto INCOPA, Centro Internacional de la Papa. Lima, Perú. 55 p.

Thiele, G., Bernet, T. (eds.). 2005. Conceptos, Pautas y Herramientas: Enfoque Participativo en Cadenas Productivas y Plataformas de Concertación. Proyecto Papa Andina, Centro Internacional de la Papa (CIP). Lima, Perú. 171 p. 